\title{
EXPANSION OF ANALYTIC FUNCTIONS OF AN OPERATOR IN SERIES OF FABER POLYNOMIALS
}

\section{Maurice Hasson}

Let $T: B \rightarrow B$ be a bounded linear operator on the complex Banach space $B$ and let $f(z)$ be analytic on a domain $D$ containing the spectrum $S_{P}(T)$ of $T$. Then $f(T)$ is defined by

$$
f(T):=\frac{1}{2 \pi i} \int_{C} f(z)(z-T)^{-1} d z
$$

where $C$ is a contour surrounding $S_{P}(T)$ and contained in $D$.

If there exists $a \in \mathbb{C}$ and $r>0$ such that the disk $|z-a|<r$ contains $S_{P}(T)$ and is contained in $D$, then

$$
f(T)=\sum_{n=0}^{\infty} a_{n}(T-a)^{n}
$$

where

$$
f(z)=\sum_{n=0}^{\infty} a_{n}(z-a)^{n}
$$

is the power series representation of $f(z)$ in $|z-a|<r$. If, however, for every disk $\Delta \subset D$, we have $\Delta \not \supset S_{P}(T)$, then the series $\sum_{n=0}^{\infty} a_{n}(T-a)^{n}$ cannot converge no matter what point $a \in D$ is chosen.

The purpose of this paper, for a given operator $T$ and function $f(z)$ whose domain $D$ contains $S_{P}(T)$, is to find an expansion

$$
f(z)=\sum_{n=0}^{\infty} a_{n} F_{n}(z)
$$

in a series of polynomials $F_{n}(z)$ where the convergence is uniform on compact subsets of some open set containing $S_{P}(T)$ and such that

$$
f(T)=\sum_{n=0}^{\infty} a_{n} F_{n}(T)
$$

where $f(T)$ is defined as above, and where the convergence takes place in the operator norm topology.

Received 21st November, 1996

The author wishes to express his appreciation to Professor Roger Nussbaum for fruitful discussions.

Copyright Clearance Centre, Inc. Serial-fee code: 0004-9729/97 \$A2.00+0.00. 


\section{INTRODUCTION}

Let $T: B \rightarrow B$ be a bounded linear operator on the complex Banach space $B$ and let $f(z)$ be analytic on a domain $D$ containing the spectrum $S_{P}(T)$ of $T$. Then $f(T)$ is defined by the well known formula

$$
f(T):=\frac{1}{2 \pi i} \int_{C} f(z)(z-T)^{-1} d z
$$

where $C$ is a contour surrounding $S_{P}(T)$ and contained in $D$.

If there exists $a \in \mathbb{C}$ and $r>0$ such that the disk $|z-a|<r$ contains $S_{P}(T)$ and is contained in $D$, then

$$
f(T)=\sum_{n=0}^{\infty} a_{n}(T-a)^{n}
$$

where

$$
f(z)=\sum_{n=0}^{\infty} a_{n}(z-a)^{n}
$$

is the power series representation of $f(z)$ in $|z-a|<r$. See [4] and [8]. However the spectral radius formula shows that the above geometric condition is also necessary:

PROPOSITION 1.1. If, for every disk $\Delta=\Delta_{a}$ of center a with $\Delta \subset D$, we have $\Delta \not \supset S_{P}(T)$, then the series $\sum_{n=0}^{\infty} a_{n}(T-a)^{n}$ cannot converge no matter what point $a \in D$ is chosen, and where the $a_{n}$ are as above.

Proof: The above hypothesis, the fact that $S_{P}(T-a)=S_{P}(T)-a$ and the Cauchy-Hadamard formula for the radius of convergence show that

$$
\|T-a\|_{S_{p}}>\frac{1}{\limsup _{n \rightarrow \infty} \sqrt[n]{\left|a_{n}\right|}}
$$

where $\|T\|_{S_{p}}$ is the spectral norm of $T$. Now the spectral radius formula

$$
\lim _{n \rightarrow \infty}\left\|T^{n}\right\|^{1 / n}=\|T\|_{S_{P}}
$$

and (1.2) yield

$$
\begin{aligned}
\limsup _{n \rightarrow \infty} \sqrt[n]{\left|a_{n}\right|}\left\|(T-a)^{n}\right\|^{1 / n} & >\frac{1}{\|T-a\|_{S_{P}}}\|(T-a)\|_{S_{P}} \\
& =1 .
\end{aligned}
$$

Relation (1.3) shows that the series

$$
\sum_{n=0}^{\infty} a_{n}(T-a)^{n}
$$

cannot converge. 
REMARK. In fact the above proof shows slightly more. (We are grateful to Michael Vogelius for having made this observation.) If $a \in D$ is such that every disk centered at $a$ and contained in $D$ does not contain $S_{P}(T)$ then the series $\sum_{n=0}^{\infty} a_{n}(T-a)^{n}$ cannot converge.

The simple example below illustrates the situation.

Let $\alpha \in \mathbb{C}, \alpha \neq 0$, and let $T: \mathbb{C}^{2} \rightarrow \mathbb{C}^{2}$ be defined by $T\left(z_{1}, z_{2}\right)=\left(-\alpha z_{1}, \alpha z_{2}\right)$ so that its matrix representation with respect to the basis $(1,0),(0,1)$ is

$$
A=\left(\begin{array}{cc}
-\alpha & 0 \\
0 & \alpha
\end{array}\right)
$$

Let $f(z)=1 / z$ and let $a \neq 0$ be a complex number. We remark that, indeed, there is no (open) disk $\Delta$ with $0 \notin \Delta$ and $-\alpha, \alpha \in \Delta$. We have

$$
\frac{1}{z}=\sum_{n=0}^{\infty}(-1)^{n} \frac{(z-a)^{n}}{a^{n+1}},|z-a|<|a|
$$

However the series

$$
\sum_{n=0}^{\infty}(-1)^{n} \frac{(A-a)^{n}}{a^{n+1}}=\frac{1}{a} \sum_{n=0}^{\infty}(-1)^{n}\left(\begin{array}{cc}
((-\alpha-a) / a)^{n} & 0 \\
0 & ((\alpha-a) a)^{n}
\end{array}\right)
$$

does not converge because $|(-\alpha-a) / a|>1$ or $|(\alpha-a) / a|>1$ as is readily verified by using the law of cosines.

The purpose of this paper, for a given operator $T$ and function $f(z)$ whose domain $D$ contains $S_{P}(T)$, is to find an expansion

$$
f(z)=\sum_{n=0}^{\infty} a_{n} F_{n}(z)
$$

in a series of polynomials $F_{n}(z)$ where the convergence is uniform on compact subsets of some open set containing $S_{P}(T)$ and such that

$$
f(T)=\sum_{n=0}^{\infty} a_{n} F_{n}(T)
$$

where $f(T)$ is defined as in (1.1), and where the convergence takes place in the operator norm topology. Moreover we shall show that in an " $N^{\text {th }}$ root sense" to be made precise later, the speed of convergence

$$
\left\|f(T)-\sum_{n=0}^{N} a_{n} F_{n}(T)\right\|
$$


where $\|$.$\| denotes the operator norm on \mathcal{L}(B)$, is dominated by

$$
\left\|f(z)-\sum_{n=0}^{N} a_{n} F_{n}(z)\right\|_{S_{P}(T)},
$$

where $\|\cdot\|_{E}$ denotes the supremum norm on the set $E \subset \mathbb{C}$.

We remark that if $T$ is a normal operator on a Hilbert space then the spectral theorem tells us that

$$
\left\|f(T)-\sum_{n=0}^{N} a_{n} F_{n}(T)\right\|=\left\|f(z)-\sum_{n=0}^{N} a_{n} F_{n}(z)\right\|_{S_{P}(T)} .
$$

To prove the same relation in an " $N^{\text {th }}$ root sense" in the case of a general operator requires the use of an appropriate contour $C$ in the complex plane in relation (1.1). This contour will happen to be the level curve of some conformal mapping, as will be shown in Section III. In that sense the proof of our main result has some similarities with the proof of the classical result $[\mathbf{4}, \mathbf{1 4}]$ that $S_{P}(T) \subset$ open left half plane $\Longrightarrow$ $\left\|e^{t T}\right\| \rightarrow 0$ as $t \rightarrow \infty$, where an appropriate contour $C$ also has to be chosen. However in the latter setting, $C$ is a contour enclosing $S_{P}(T)$ and bounded away from the $y$-axis, and conformal mapping plays no role.

The polynomials $F_{n}(z)$ happen to be the Faber polynomials for (an appropriate set $E$ containing) $S_{P}(T)$ and the next section is devoted to the review of these polynomials. Section III contains the statement and proof of our main result. The last section is devoted to illustrative examples.

\section{Preliminaries from approximation theory in the complex Plane}

In this section we state, mostly without proof, results of complex approximation theory which will be needed in the next section. We follow essentially three sources: $[11,12,13]$.

Let $E$ be a compact simply connected set of the complex plane containing more than one point and let $\omega=\phi(z)$ map conformally $\operatorname{Ext}(E)$ into $|\omega|>1$ and with $\phi(\infty)=\infty$. The map $\phi(z)$ has the form

$$
\phi(z)=\frac{z}{c}+a_{0}+\frac{a_{-1}}{z}+\frac{a_{-2}}{z^{2}}+\ldots .
$$

The number $c>0$ is the capacity $\operatorname{Cap}(E)$ of the set $E$. The Faber polynomials for $E$, $F_{n}(z)$, consist of the polynomial part of $\phi(z)^{n}$. The inverse map is denoted $z=\psi(\omega)$ and has the form

$$
\psi(\omega)=c \omega+b_{0}+\frac{b_{-1}}{\omega}+\frac{b_{-2}}{\omega^{2}}+\ldots
$$


For $\rho>1$ let $\Gamma_{\rho}$ be the level curve

$$
\Gamma_{\rho}:=\{z \in \mathbb{C} ;|\phi(z)|=\rho\} .
$$

As an example consider $E=[-1,1]$. Then

$$
\omega=\phi(z)=z+\sqrt{z^{2}-1}=\frac{z}{\frac{1}{2}}-\frac{1}{2 z}-\frac{1}{8 z^{3}}+\ldots
$$

and

$$
z=\psi(\omega)=\frac{1}{2} \omega+\frac{1}{2 \omega} .
$$

Hence $\Gamma_{\rho}(\rho>1)$ is the ellipse with foci $[-1,1]$ and $\rho+1 / \rho,(\rho-1 / \rho)$ for lengths of its axes. The Faber polynomials for $[-1,1]$ are $F_{n}(z)=2 T_{n}(z)$ where $T_{n}(x)=$ $\cos (n \arccos x)$ are the Chebyshev polynomials. (See the remark following the proof of Walsh's theorem below.)

If the mapping function extends continuously and in an bijective manner to the boundary $\mathrm{B}_{r}(E)$ of $E$, which is the case if $\mathrm{B}_{r}(E)$ is a Jordan curve, we write $\Gamma_{1}=$ $\mathrm{B}_{r}(E)$. In that case the mapping function and its inverse are still denoted by $\omega=\phi(z)$ and $z=\psi(\omega)$ respectively.

The following celebrated theorem of Walsh [13] will play a fundamental role in the sequel.

ThEOREM 2.1. (Walsh) Let $E$ be as above and let $P_{n}(z)$ be a polynomial of degree (at most) $n$ with

$$
\left\|P_{n}(z)\right\|_{E} \leqslant 1
$$

Then, for $\rho>1$, one has

$$
\left\|P_{n}(z)\right\|_{\Gamma_{\rho}} \leqslant \rho^{n}
$$

Because of the importance of this theorem in our work, we give a simplified proof of it made possible by the additional assumption that $\mathrm{B}_{r}(E)$ is a Jordan curve. In that case the maximum principle extends to the extended mapping function.

Proof: Because the Laurent expansion at $\omega=\infty$ of $z=\psi(\omega)$ is of the form $\psi(\omega)=c \omega+b_{0}+\left(b_{-1} / \omega\right)+\left(b_{-2} / \omega^{2}\right)+\ldots$, the function $P_{n}(\psi(\omega))$ has a pole of order $n$ at $\infty$. It follows that

$$
f(\omega):=\frac{P_{n}(\psi(\omega))}{\omega^{n}}
$$

is analytic at $\infty$. Now the maximum principle yields, for $\rho \geqslant 1$,

$$
\sup _{|\omega|=1}\left|\frac{P_{n}(\psi(\omega))}{\omega^{n}}\right| \geqslant \sup _{|\omega|=\rho}\left|\frac{P_{n}(\psi(\omega))}{\omega^{n}}\right| .
$$


Walsh's theorem follows if we remark that

$$
\sup _{|\omega|=\rho}\left|P_{n}(\psi(\omega))\right|=\sup _{z \in \Gamma_{\rho}}\left|P_{n}(z)\right|
$$

For a proof of Walsh's theorem in which the hypothesis that $\mathrm{B}_{r}(E)$ is a Jordan curve is deleted, see [11].

Notice that we deviate slightly from the usual definition of the Faber polynomials in the sense that our function $\omega=\phi(z)$ maps $\operatorname{Ext}(E)$ onto $|\omega|>1$ instead of $|\omega|>c$, where $c=\operatorname{Cap}(E)$, as it is usually the case. It follows that the Faber polynomials as often described in the literature correspond to $c^{n} F_{n}(z)$ here. In particular the Faber polynomials for $[-1,1]$ are usually $\left(1 / 2^{n-1}\right) T_{n}(z)$ instead of $2 T_{n}(z)$. We found it more convenient to make this modification. For the same reason, our statement of Walsh's theorem differs from that in [11]. Note that some authors, like Curtiss in [3], use the convention adopted here.

Let $z \in \operatorname{Ext}(E)$ and let $\rho>1$ be such that $z \in \Gamma_{\rho}$. Then for $|\omega|>\rho$ the following generating function for the Faber polynomials

$$
\frac{\psi^{\prime}(\omega)}{\psi(\omega)-z}=\sum_{n=0}^{\infty} \frac{F_{n}(z)}{\omega^{n+1}}
$$

holds where the convergence is uniform in the compact subsets of $|\omega|>\rho$.

One of the important consequences of this fundamental relation is the following expansion of analytic functions in series of Faber polynomials:

THEOREM 2.2. Let $f(z)$ be analytic in a domain $D$ containing $E$ and let $F_{n}(z)$ be the Faber polynomials for $E$. Let $\rho>1$ be such that $\Gamma_{\rho} \subset D$. Then

$$
f(z)=\sum_{n=0}^{\infty} a_{n} F_{n}(z)
$$

where

$$
a_{n}:=\frac{1}{2 \pi i} \int_{C^{\prime}} \frac{f(\psi(\omega))}{\omega^{n+1}} d \omega
$$

and where $C^{\prime}$ is the image by $\omega=\phi(z)$ of a closed curve $C$ contained in $D$ and not intersecting $E$. Moreover the convergence is uniform in the compact subsets of Int $\left(\Gamma_{\rho^{\prime}}\right)$ where $\rho^{\prime}$ is the supremum of the numbers $\rho$ such that $\operatorname{Int}\left(\Gamma_{\rho}\right) \subset D$.

As well known, under mild conditions on $\mathrm{B}_{r}(E)$, the partial sums $\sum_{n=0}^{N} a_{n} F_{n}(z)$ are, up to a factor of $K \log (N)$, the best approximation of $f(z)$ by polynomials of degree $N$ for the supremum norm on $E$. See $[3,7,9]$. The following specialised version of Bernstein's theorem [11] will be needed. 
Proposition 2.3. Let $E$ and $\rho^{\prime}$ be as above. Then

$$
\underset{N \rightarrow \infty}{\limsup }\left\|f(z)-\sum_{n=0}^{N} a_{n} F_{n}(z)\right\|_{E}^{1 / N}=\frac{1}{\rho^{\prime}}
$$

Proof: It follows easily from $(2.1)$, by taking $C=\{|\omega|=\rho\}, \rho<\rho^{\prime}$, that

$$
\limsup _{n \rightarrow \infty}\left|a_{n}\right|^{1 / n} \leqslant \frac{1}{\rho^{\prime}}
$$

On the other hand (see [9])

$$
\left\|F_{n}(z)\right\|_{E} \leqslant n^{\alpha}
$$

for some $\alpha$. It readily follows that

$$
\underset{N \rightarrow \infty}{\limsup }\left\|\sum_{n=N+1}^{\infty} a_{n} F_{n}(z)\right\|_{E}^{1 / N} \leqslant \frac{1}{\rho^{\prime}} .
$$

That is to say

$$
\limsup _{N \rightarrow \infty}\left\|f(z)-\sum_{n=0}^{N} a_{n} F_{n}(z)\right\|_{E}^{1 / N} \leqslant \frac{1}{\rho^{\prime}} .
$$

Equality above follows from standard overconvergence arguments. Indeed if strict inequality was to prevail above, then $f(z)$ would be analytic in a domain containing Int $\Gamma_{\rho^{\prime \prime}}$ with $\rho^{\prime \prime}>\rho^{\prime}$, contrarily to the definition of $\rho^{\prime}$.

\section{Statement and Proof of the Main Result}

Let $T: B \rightarrow B$ be a bounded linear operator on the complex Banach space $B$ and let $f(z)$ be analytic on a domain $D$ containing $S_{P}(T)$. In what follows we assume that $S_{P}(T)$ is a connected (compact) set and we designate by $E$ the smallest (compact) simply connected set containing $S_{P}(T)$. The non extended mapping functions $\omega=\phi(z)$ and $z=\psi(\omega)$ are as described in Section II. Remark that $\|f(z)\|_{S_{P}(T)}=\|f(z)\|_{E}$. Our main results states:

THEOREM 3.1. Let $f(z)$ be analytic on a domain $D$ containing $E$ and let $F_{n}(z)$ be the Faber polynomials for $E$. Let, with $a_{n}$ given in (2.1),

$$
f(z)=\sum_{n=0}^{\infty} a_{n} F_{n}(z)
$$

be the expansion of $f(z)$ in Faber polynomials. Then

$$
f(T)=\sum_{n=0}^{\infty} a_{n} F_{n}(T)
$$


where

$$
f(T):=\frac{1}{2 \pi i} \int_{C} f(z)(z-T)^{-1} d z .
$$

Moreover let $\rho>1$ be such that $\Gamma_{\rho} \subset D$. Then

$$
\limsup _{N \rightarrow \infty}\left\|f(T)-\sum_{n=0}^{N} a_{n} F_{n}(T)\right\|^{1 / N} \leqslant \frac{1}{\rho} .
$$

If, in addition, the number $\rho$ has the following property:

$$
\delta>\rho \Longrightarrow \operatorname{Int}\left(\Gamma_{\delta}\right) \not \subset D
$$

then

$$
\begin{aligned}
\limsup _{N \rightarrow \infty}\left\|f(T)-\sum_{n=0}^{N} a_{n} F_{n}(T)\right\|^{1 / N} & =\limsup _{N \rightarrow \infty}\left\|f(z)-\sum_{n=0}^{N} a_{n} F_{n}(z)\right\|_{S_{P}(T)}^{1 / N} \\
& =\frac{1}{\rho} .
\end{aligned}
$$

Remark. Theorem 2.2 and Proposition 2.3, together with Walsh's theorem and an appropriate contour $C$, can be used to show, by following an argument similar to that of Lemma 3.2 below, that the partial sums of $\sum_{n=0}^{\infty} a_{n} F_{n}(T)$ form a Cauchy sequence in $\mathcal{L}(B)$. Part of the work below consists precisely of proving that the sum is $f(T)$ as defined in (1.1).

The Proof of Theorem 3.1 relies on several preparatory results.

LEMMA 3.2. Let $P_{n}(z)$ be a sequence of polynomials each of degree (at most) $n$ with the following property:

$$
\limsup _{n \rightarrow \infty}\left\|P_{n}(z)\right\|_{E}^{1 / n}=1
$$

Then

$$
\sum_{n=0}^{\infty} \frac{P_{n}(T)}{\omega^{n+1}}
$$

converges uniformly on the compact subsets of $|\omega|>1$.

PROOF: In what follows $M$ is a generic constant whose value may change from occurrence to occurrence. Let $\varepsilon>0$. Then, for $n$ big enough,

$$
\left\|P_{n}(z)\right\|_{E} \leqslant(1+\varepsilon)^{n}
$$


Now

$$
P_{n}(T)=\frac{1}{2 \pi i} \int_{\Gamma_{1+\varepsilon}} P_{n}(z)(z-T)^{-1} d z .
$$

The fact that the curve $\Gamma_{1+\varepsilon}$ is bounded away from $S_{P}(T)$ and standard continuity (the curve $\Gamma_{1+\varepsilon}$ is analytic) and compactness arguments, together with Walsh's theorem, yield

$$
\sup _{t}\left(\left|P_{n}(\gamma(t))\right|\left\|(\gamma(t) I-T)^{-1}\right\|\left|\gamma^{\prime}(t)\right|\right) \leqslant M(1+\varepsilon)^{2 n}
$$

where $\gamma(t)$ is a $C^{1}$ parametrisation of $\Gamma_{1+\varepsilon}$. Hence

$$
\left\|P_{n}(T)\right\| \leqslant M(1+\varepsilon)^{2 n} .
$$

It follows, because $\varepsilon>0$ is arbitrary, that

$$
\limsup _{n \rightarrow \infty}\left\|P_{n}(T)\right\|^{1 / n} \leqslant 1
$$

In fact the above inequality, the hypothesis $\limsup _{n \rightarrow \infty}\left\|P_{n}(z)\right\|_{E}^{1 / n}=1$ and the inequality $\left\|P_{n}(z)\right\|_{E} \leqslant\left\|P_{n}(T)\right\|$ imply

$$
\limsup _{n \rightarrow \infty}\left\|P_{n}(T)\right\|^{1 / n}=1
$$

It follows that

$$
\sum_{n=0}^{\infty} \frac{P_{n}(T)}{\omega^{n+1}}
$$

converges uniformly on the compact subsets of $|\omega|>1$ as was to be proved.

LEMMA 3.3.

$$
\omega \mapsto \omega \psi^{\prime}(\omega)(\psi(\omega)-T)^{-1}
$$

is analytic at $\infty$.

PROOF: Remark first that $\psi(\omega)$ is defined for $|\omega|>1$ so that $\psi(\omega) \notin E \supset S_{P}(T)$. Hence $\psi(\omega)-T$ is invertible and bounded. The term in the highest power of $\omega$ of the asymptotic expansion at $\infty$ of $\omega \psi^{\prime}(\omega)(\psi(\omega)-T)^{-1}$ is $c \omega(c \omega-T)^{-1}$ where $c$ is the capacity of $E$. But

$$
c \omega(c \omega-T)^{-1}=I+\frac{T}{c \omega}+\ldots
$$

at $\infty$. This shows that the asymptotic expansion

$$
\omega \psi^{\prime}(\omega)(\psi(\omega)-T)^{-1}=I+\frac{T}{c \omega}+\ldots
$$

is valid at $\infty$.

Hence Lemma 3.3 follows. 
REMARK. What will be used in the proof of Proposition 3.4 below is the fact that the map

$$
\omega \mapsto \omega \psi^{\prime}(\omega)\left\langle\mathcal{X},(\psi(\omega)-T)^{-1}\right\rangle
$$

is analytic at $\infty$ where $\mathcal{X} \in(\mathcal{L}(B))^{\prime}$, the dual of $\mathcal{L}(B)$. This follows at once from Lemma 3.3 and the continuity and linearity of $\mathcal{X}$.

Lemmas 3.2 and 3.3, in conjunction with the Hahn-Banach theorem and the observation below, will allow us to prove the following extension of the generating function of the Faber polynomials. As already noticed, $\left\|F_{n}(z)\right\|_{E} \leqslant n^{\alpha}$ for some $\alpha$. It then follows that $\limsup _{n \rightarrow \infty}\left\|F_{n}(z)\right\|_{E}^{1 / n}=1$. This result also follows from the well known representation formula of $F_{n}(z)$ in term of $\phi(z)$ :

$$
F_{n}(z)=\frac{1}{2 \pi i} \int_{C} \frac{\phi(\xi)^{n}}{\xi-z} d \xi
$$

(This also shows that $\left\|F_{n}(z)\right\|_{E} \leqslant M$ if the boundary $B_{r}(E)$ of $E$ is an analytic curve. See [6].) As in the proof of the usual generating function formula, the following observation will play a central role.

From the definition of the Faber polynomials $F_{n}(z)$ we see that

$$
\xi \mapsto \frac{\phi(\xi)^{n}-F_{n}(\xi)}{\xi-z}
$$

has a zero of order two at $\infty$.

Proposition 3.4 .

$$
\psi^{\prime}(\omega)(\psi(\omega)-T)^{-1}=\sum_{n=0}^{\infty} \frac{F_{n}(T)}{\omega^{n+1}}
$$

holds where the convergence is uniform in the compact subsets of $|\omega|>1$.

PROOF: Let $\bar{C}$ be a contour containing $E$ in its interior, and let $C$ be a contour with $C \subset \operatorname{Int}(\bar{C}), \operatorname{Int}(C) \supset E$. The above observation yields,

$$
\frac{1}{2 \pi i} \int_{C} \frac{1}{2 \pi i} \int_{\bar{C}} \frac{\phi(\xi)^{n}-F_{n}(\xi)}{\xi-z} d \xi(z-T)^{-1} d z=0 .
$$

Indeed the inner integral above vanishes in view of the zero of order two at $\xi=\infty$ of $\xi \mapsto\left(\phi(\xi)^{n}-F_{n}(\xi)\right) /(\xi-z)$ and the fact that $z \in C \subset \operatorname{Int}(\bar{C})$. Now

$$
\begin{aligned}
\frac{1}{2 \pi i} \int_{C} \frac{1}{2 \pi i} \int_{\bar{C}} \frac{F_{n}(\xi)}{\xi-z} d \xi(z-T)^{-1} d z & =\frac{1}{2 \pi i} \int_{C} F_{n}(z)(z-T)^{-1} d z \\
& =F_{n}(T) .
\end{aligned}
$$


On the other hand

$$
\begin{aligned}
\frac{1}{2 \pi i} \int_{C} \frac{1}{2 \pi i} \int_{\bar{C}} \frac{\phi(\xi)^{n}}{\xi-z} d \xi(z-T)^{-1} d z & =\frac{1}{2 \pi i} \int_{C} \frac{1}{2 \pi i} \int_{C^{\prime}} \frac{\omega^{n-1} \omega \psi^{\prime}(\omega)}{\psi(\omega)-z} d \omega(z-T)^{-1} d z \\
& =\frac{1}{2 \pi i} \int_{C^{\prime}} \omega^{n-1} \omega \psi^{\prime}(\omega)(\psi(\omega)-T)^{-1} d \omega
\end{aligned}
$$

where $C^{\prime}$ is the image by $\omega=\phi(z)$ of $\bar{C}$. Hence (3.3), (3.4) and (3.5) yield

$$
F_{n}(T)=\frac{1}{2 \pi i} \int_{C^{\prime}} \omega^{n-1} \omega \psi^{\prime}(\omega)(\psi(\omega)-T)^{-1} d \omega
$$

Let now $\mathcal{X} \in(\mathcal{L}(B))^{\prime}$, the dual of $\mathcal{L}(B)$. We have, using the continuity and linearity of the integral and $\mathcal{X}$,

$$
\left\langle\mathcal{X}, F_{n}(T)\right\rangle=\frac{1}{2 \pi i} \int_{C^{\prime}} \omega^{n-1} \omega \psi^{\prime}(\omega)\left\langle\mathcal{X},(\psi(\omega)-T)^{-1}\right\rangle d \omega .
$$

This shows that $\left\langle\mathcal{X}, F_{n}(T)\right\rangle$ is the coefficient of $\omega^{-n}$ in the Laurent expansion at $\omega=\infty$ of

$$
\omega \mapsto \omega \psi^{\prime}(\omega)\left\langle\mathcal{X},(\psi(\omega)-T)^{-1}\right\rangle
$$

Now by (the remark following) Lemma 3.3 we have that $\omega \psi^{\prime}(\omega)\left\langle\mathcal{X},(\psi(\omega)-T)^{-1}\right\rangle$ is analytic at $\infty$ so that its Laurent expansion at $\infty$ contains no positive powers of $\omega$. That is to say

$$
\sum_{n=0}^{\infty} \frac{\left\langle\mathcal{X}, F_{n}(T)\right\rangle}{\omega^{n}}=\omega \psi^{\prime}(\omega)\left\langle\mathcal{X},(\psi(\omega)-T)^{-1}\right\rangle .
$$

Now using again the continuity and linearity of $\mathcal{X}$ and the fact that, by Lemma 3.2 , $\sum_{n=0}^{\infty}\left(F_{n}(T)\right) /\left(\omega^{n+1}\right)$ converges uniformly on the compact subsets of $|\omega|>1$, we obtain

$$
\left\langle\mathcal{X}, \sum_{n=0}^{\infty} \frac{F_{n}(T)}{\omega^{n}}\right\rangle=\left\langle\mathcal{X}, \omega \psi^{\prime}(\omega)(\psi(\omega)-T)^{-1}\right\rangle
$$

Hence the Hahn-Banach theorem tells us, because $\mathcal{X} \in(\mathcal{L}(B))^{\prime}$ is arbitrary, that

$$
\sum_{n=0}^{\infty} \frac{F_{n}(T)}{\omega^{n+1}}=\psi^{\prime}(\omega)(\psi(\omega)-T)^{-1}
$$

where the convergence is uniform in the compact subsets of $|\omega|>1$, again because $\sum_{n=0}^{\infty}\left(F_{n}(T) / \omega^{n+1}\right)$ converges uniformly on the compact subsets of $|\omega|>1$, as was to be proved. 
REMARK. Proposition 3.4 also shows that $F_{n}(T)$ is the coefficient of $\omega^{-n-1}$ in the Laurent expansion at $\infty$ of

$$
\psi^{\prime}(\omega)(\psi(\omega)-T)^{-1}
$$

Proposition 3.4 allows us now to expand $f(T)$ in series of Faber polynomials in $T$.

THEOREM 3.5. Let $f(z)$ be analytic in a domain $D$ containing $S_{P}(T)$, let $E$ be as above and let $F_{n}(z)$ be the Faber polynomials for $E$. Then

$$
f(T)=\sum_{n=0}^{\infty} a_{n} F_{n}(T)
$$

where

$$
f(T):=\frac{1}{2 \pi i} \int_{C} f(z)(z-T)^{-1} d z
$$

and

$$
a_{n}:=\frac{1}{2 \pi i} \int_{C^{\prime}} \frac{f(\psi(\omega))}{\omega^{n+1}} d \omega
$$

and where the convergence takes place in $\mathcal{L}(B)$.

Proof: With $\bar{C}, C$ and $C^{\prime}$ as in the proof of Proposition 3.4, with the additional assumption that $\bar{C} \subset D$,

$$
\begin{aligned}
f(T) & =\frac{1}{2 \pi i} \int_{C} f(z)(z-T)^{-1} d z \\
& =\frac{1}{2 \pi i} \int_{C} \frac{1}{2 \pi i} \int_{\bar{C}} \frac{f(\xi)}{\xi-z} d \xi(z-T)^{-1} d z \\
& =\frac{1}{2 \pi i} \int_{C} \frac{1}{2 \pi i} \int_{C^{\prime}} \frac{f(\psi(\omega)) \psi^{\prime}(\omega)}{\psi(\omega)-z} d \omega(z-T)^{-1} d z \\
& =\frac{1}{2 \pi i} \int_{C^{\prime}} f(\psi(\omega)) \psi^{\prime}(\omega)(\psi(\omega)-T)^{-1} d \omega \\
& =\frac{1}{2 \pi i} \int_{C^{\prime}} f(\psi(\omega)) \sum_{n=0}^{\infty} \frac{F_{n}(T)}{\omega^{n+1}} d \omega
\end{aligned}
$$

where the last equality is obtained from Proposition 3.4. It follows that

$$
f(T)=\sum_{n=0}^{\infty} a_{n} F_{n}(T)
$$

where

$$
a_{n}:=\frac{1}{2 \pi i} \int_{C^{\prime}} \frac{f(\psi(\omega))}{\omega^{n+1}} d \omega
$$


and where the interchange of the integral with the summation is justified in view of the uniform convergence of

$$
\sum_{n=0}^{\infty} \frac{F_{n}(T)}{\omega^{n+1}}
$$

on the compact subsets of $|\omega|>1$.

We now have built the necessary tools for the proof of our main result, Theorem 3.1. In view of Theorem 3.5, only the error estimates remain to be proved. We prove only (3.2) because this relation clearly implies (3.1).

PROOF OF THEOREM 3.1: We remark that in the formula

$$
a_{n}=\frac{1}{2 \pi i} \int_{|\omega|=\rho^{\prime}} \frac{f(\psi(\omega))}{\omega^{n+1}} d \omega
$$

$\rho^{\prime}$ may be taken as close as we please to $\rho$ as far as $\rho^{\prime}<\rho$. It follows easily that

$$
\limsup _{n \rightarrow \infty}\left|a_{n}\right|^{1 / n} \leqslant \frac{1}{\rho}
$$

In fact we have

$$
\limsup _{n \rightarrow \infty}\left|a_{n}\right|^{1 / n}=\frac{1}{\rho}
$$

Indeed overconvergence (in the sense of Walsh) arguments show that $\limsup _{n \rightarrow \infty}\left|a_{n}\right|^{1 / n}<$ $1 / \rho$ implies $f(z)$ is analytic in $\Gamma_{\delta}$ with $\delta>\rho$, which is not possible in view of the definition of $\rho$. Now $\limsup _{n \rightarrow \infty}\left|a_{n}\right|^{1 / n}=1 / \rho$ implies

$$
\limsup _{N \rightarrow \infty}\left(\sum_{n=N}^{\infty}\left|a_{n}\right|\right)^{1 / N}=\frac{1}{\rho}
$$

On the other hand

$$
\limsup _{n \rightarrow \infty}\left\|F_{n}(z)\right\|_{E}^{1 / n}=1
$$

It follows from $(3.6),(3.7)$ and standard estimates that

$$
\limsup _{N \rightarrow \infty}\left\|f(T)-\sum_{n=0}^{N} a_{n} F_{n}(T)\right\|^{1 / N} \leqslant \frac{1}{\rho} .
$$

But

$$
\left\|f(T)-\sum_{n=0}^{N} a_{n} F_{n}(T)\right\| \geqslant\left\|f(z)-\sum_{n=0}^{N} a_{n} F_{n}(z)\right\|_{S_{P}(T)} .
$$


We obtain, using the above overconvergence arguments and Proposition 2.3,

$$
\underset{N \rightarrow \infty}{\limsup }\left\|f(z)-\sum_{n=0}^{N} a_{n} F_{n}(z)\right\|_{S_{P}(T)}^{1 / N}=\frac{1}{\rho} .
$$

It follows from (3.8), (3.9) and (3.10) that

$$
\begin{aligned}
\underset{N \rightarrow \infty}{\limsup }\left\|f(T)-\sum_{n=0}^{N} a_{n} F_{n}(T)\right\|^{1 / N} & =\limsup _{N \rightarrow \infty}\left\|f(z)-\sum_{n=0}^{N} a_{n} F_{n}(z)\right\|_{S_{P}(T)}^{1 / N} \\
& =\frac{1}{\rho}
\end{aligned}
$$

as was to be shown.

The proof of Theorem 3.1 is complete.

\section{Concluding Remarks}

Theorem 3.1 also shows that

$$
\lim _{N \rightarrow \infty}\left\|f(T)-\sum_{n=0}^{N} a_{n} F_{n}(T)\right\|^{1 / N}=0
$$

if and only if $f(z)$ is entire.

We restricted ourselves to the case when $S_{P}(T)$ is connected, however this constraint is unnecessary. It suffices to take for $E$ a compact simply connected set containing $S_{P}(T)$ and containing $D$. However in that case conclusion (3.2) of Theorem 3.1 will have to be replaced by

$$
\begin{aligned}
\underset{N \rightarrow \infty}{\limsup }\left\|f(T)-\sum_{n=0}^{N} a_{n} F_{n}(T)\right\|^{1 / N} & \leqslant \limsup _{N \rightarrow \infty}\left\|f(z)-\sum_{n=0}^{N} a_{n} F_{n}(z)\right\|_{E}^{1 / N} \\
& =\frac{1}{\rho}
\end{aligned}
$$

which is weaker than (3.2) because now $\|f(z)\|_{E} \geqslant\|f(z)\| s p(T)$.

Even if there exists a disk $\Delta$ of center $a$ with $D \supset \Delta \supset S_{P}(T)$, so that expansion of $f(T)$ in series of monomials $(T-a)^{n}$ is possible, it may be advantageous to expand $f(T)$ in series of Faber polynomials as the simple example below illustrates. (For the problem of numerical evaluation of the Faber polynomials see $[\mathbf{1}, \mathbf{2}, \mathbf{5}]$ and the references therein.) 
Let $T$ with $S_{P}(T)=[-1,1]$ and let $f(z)=1 /(z-2)$. Then

$$
f(T)=-\sum_{n=0}^{\infty} \frac{T^{n}}{2^{n+1}}
$$

and

$$
\limsup _{N \rightarrow \infty}\left\|f(T)+\sum_{n=0}^{N} \frac{T^{n}}{2^{n+1}}\right\|^{1 / N}=\frac{1}{2}
$$

The expansion of $f(T)$ in series of Faber polynomials for $[-1,1]$ is

$$
f(T)=\sum_{n=0}^{\infty} a_{n} F_{n}(T)
$$

where

$$
a_{n}=\frac{1}{2 \pi i} \int_{C} \frac{f(\psi(\omega))}{\omega^{n+1}} d \omega
$$

with

$$
\psi(\omega)=\frac{1}{2} \omega+\frac{1}{2 \omega}
$$

and

$$
F_{n}(z)=2 T_{n}(z) .
$$

(The $a_{n}$ are half the Fourier coefficients of $1 /(\cos \theta-2)$. See [10].) Now the level curve which passes through the point 2 is the ellipse $\Gamma_{2+\sqrt{3}}$. It follows by Theorem 3.1 that

$$
\limsup _{N \rightarrow \infty}\left\|f(T)-\sum_{n=0}^{N} a_{n} F_{n}(T)\right\|^{1 / N}=\frac{1}{2+\sqrt{3}}
$$

Comparison of (4.1) with (4.2) shows a faster rate of convergence when the expansion in series of Faber polynomials is used.

Let now $f(z)=1 /\left(z^{2}+1 / 4\right)$ and $T$ be as above. Then, in view of Proposition 1.1, expansion of $f(T)$ in series of monomials $(T-a)^{n}$ is not possible whereas expansion in series of Faber polynomials for $[-1,1]$ gives

$$
\limsup _{N \rightarrow \infty}\left\|f(T)-\sum_{n=0}^{N} a_{n} F_{n}(T)\right\|^{1 / N}=\frac{2}{1+\sqrt{5}}
$$

if we remark that the level curve $\Gamma_{(1+\sqrt{5}) / 2}$ passes through the points $i / 2$ and $-i / 2$. 


\section{REFERENCES}

[1] J.P. Coleman and N.J. Myers, 'The Faber Polynomials for Annular Sectors', Math. Comp. 64 (1995), 181-203.

[2] J.P. Coleman and R.A. Smith, 'The Faber polynomials for circular sectors', Math. Comp. 49 (1987), 231-241.

[3] J.H. Curtiss, 'Faber polynomials and the Faber series', Amer. Math. Monthly 78 (1971), $577-596$.

[4] N. Dunford and J. Schwartz, Linear operators 1 (Interscience Publishers, New York, 1958).

[5] S.W. Ellacott, 'A survey of Faber methods in numerical approximation', Comput. Math. Appl. 12 (1986), 1103-1107.

[6] M. Hasson and B. Walsh, 'Singular points of analytic functions expanded in series of Faber polynomials' (to appear).

[7] P. Henrici, Applied and computational complex analysis 3 (Wiley, New York, 1986).

[8] R. Kadison and J. Ringrose, Fundamentals of the theory of operator algebras 1 (Academic Press, New York, 1983).

[9] T. Kövari and C. Pommerenke, 'On Faber polynomials and Faber expansions', Math. Z. 99 (1967), 193-206.

[10] G.G. Lorentz, Approximation of functions (Holt, Rinehart and Winston, New York, 1966).

[11] A.I. Markushevich, Theory of functions of a complex variable 3 (Chelsea Publishing Co., New York, 1977).

[12] V.I. Smirnov and N.A. Lebedev, Functions of a complex variable (The M.I.T. Press, Cambridge, 1968).

[13] J. Walsh, Interpolation and approximation by rational functions in the complex domain, (5th edition) (American Mathematical Society, Providence, RI, 1969).

[14] K. Yoshida, Functional analysis, (6th edition) (Springer Verlag, Berlin, Heidelbrg, New York, 1980).

Department of Mathematics

Rutgers University

New Brunswick NJ 08903

United States of America

e-mail: hasson@math.rutgers.edu 